\title{
Novel Implementation of Multiple Automated Ground Vehicles Traffic Real Time Control Algorithm for Warehouse Operations: Djikstra Approach
}

\author{
Pasan Dharmasiri \\ School of Science and Technology (SST), \\ RMIT University, Ho Chi Minh City, Vietnam \\ E-mail: S3747838@ rmit.edu.vn (Corresponding author)
}

Ilya Kavalchuk

Lead Technical Specialist, Hyperspeed Transportation System,

Sky Way, Minsk, Belarus

E-mail: ikavalch@gmail.com

\author{
Mohammadreza Akbari \\ School of Business \& Management (SBM), \\ RMIT University, Ho Chi Minh City, Vietnam \\ E-mail: reza.akbari@rmit.edu.vn
}

\begin{abstract}
Automated Guided Vehicle (AGV) systems have benefited numerous industrial warehouses in the transportation of components, parts, and raw materials. Although the AGV systems have offered great flexibility, the implementation of AGV systems imposes major challenges in the management of traffic between multiple ground vehicles inside the warehouse. An AGV traffic control system in conjunction with an anticollision path planning algorithm presents efficiencies for transferring parts in the warehouse operations. This paper evaluates several path planning algorithms and traffic control algorithms that can be implemented with multiple ground vehicles. The application of the Dijkstra approach is proposed as the most efficient traffic control algorithm and path planning algorithm with the implementation of the anticollision algorithm. The suggested algorithm is simulated using MATLAB software to check its rationality and performances under a real-life scenario and for comparison with the alternatives. The traffic control algorithm for multiple AGV systems has been performed in a dynamic environment and a time-based simulation and calculations have been used to optimize the velocity profile for each AGV. The finding from this paper presents timely and valuable insights into smart warehouses and logistics phenomenon, as a potential mechanism for optimizing material handling in warehouse management to be more efficient and collision-free through the use of modern technologies such as AGV systems and Industry 4.0 integration.
\end{abstract}

Keywords: $A G V$ system, anti-collision algorithm, industry 4.0, supply chain management, traffic control, warehouse automation, smart logistics, smart warehouse

\section{INTRODUCTION}

With the rapid expansion of the supply chain and globalization, it is important to address the rising complexity of material handling in warehouse operations. As a result of this immediate global growth in the supply chain and fierce competition (Akbari, 2013; Mujkić, Qorri, \& Kraslawksi, 2018), efficient operations, transportation and warehousing have given a substantial interest amongst companies (König $\&$ Spinler, 2016). With the shortage of labor and increasing retailers offering fast delivery options, the process in a warehouse is getting more complicated over time (Kasemset $\&$ Sudphan, 2014). The rapid technology development presents companies with a variety of choices for the moving of components, parts and raw materials, especially inside the warehouses. Automating the warehouses would increase the efficiency of the warehouse operations and support more efficient integration of the fourth industrial revolution through improved automation. According to Robotics Business Review (2019), a research report by LogisticsIQ estimates more than two times increments of the global robotic warehouses from US\$13 Billion in the year 2018 up to more than US\$27 Billion by 2025 .

In the current industry, most of the task has been automated to increase the efficiency of supply chain operations. To acquire the maximum efficiency from automation, this implementation needs to be synchronized and establish a common form of communication. The fourth industrial revolution, Industry 4.0, has been developed through this era with the growing number of computers, digitization and devices getting interconnected in the autonomous global systems to peaks its maximum efficiency on all possible levels (Tjahjono, Esplugues, Ares, \& Pelaez, 2017). The productivity also improved by enabling flexible 
manufacturing, analytical and cognitive systems with reduced over manufacturing and strict timing of all processes (Jazdi, 2016; Park, Crespi, Park, \& Kim, 2014).

Due to the increase in supply chain complexity (Akbari \& Hopkins, 2016; Christopher, 2016), warehouse operations need to be automated to increase the efficiency of those operations. Automated guided vehicles (AGV) widely used in many fully automated and semi-automated warehouses to automate the object transferring services inside the warehouse (Balasooriya, Chi, \& Kavalchuk, 2019; S.-n. Liu, 2008). However, most of the current warehouses are large in scale, implementing a single AGV is insufficient to complete the task inside the warehouse and to achieve required performance indicators. Therefore, the implementation of the Multi-AGV system is required to reduce complexity and the manual workload of a warehouse (Petrinec, Kovacic, \& Marozin, 2003). Additionally, managing the AGVs in the Multi-AGV system to become conflict-free and collisionfree is the most challenging task (Smolic-Rocak, Bogdan, Kovacic, \& Petrovic, 2010) which needs to be solved while implementing the multi-AGV system in a warehouse.

In the current developing market, most warehouses are still in the process of developing toward fully automated solutions. Therefore, the AGV system needs to be defined as a semi-automated warehouse that involves human interaction in its operations (Oleari, Magnani, Ronzoni, \& Sabattini, 2014). Furthermore, most recent research projects proposed AGV traffic control systems for fully automated warehouses that cannot implement on semi-automated warehouses in developing countries like Vietnam (S.-n. Liu, 2008).

AGVs are widely used in most of the industrial warehouse to automate the materials and goods handling process. Implementing Multiple AGV system is challenging since the multiple AGV system requires to have an integrated traffic control algorithm to eliminate collisions between AGVs in a dynamic environment (Z. Zhang, Guo, Chen, \& Yuan, 2018).

Traffic control algorithms that can be implemented to a multiple AGV system are proposed in many existing research works (Noh \& Yoon, 2016; Secchi, Olmi, Rocchi, \& Fantuzzi, 2015; W. Zhang, Peng, Wei, \& Kou, 2018; Yingfeng Zhang, Zhu, \& Lv, 2017). Most of these proposed algorithms are defined as a static environment and can only be implemented for a fully automated system. The alternative in Fang et al. (2018); Lin, Yuan, Shi, and Wang (2017); C. Liu, Tan, Zhao, Li, and Bai (2017) proposed reliable algorithm, which requires high computational power. Alternative from Digani, Sabattini, Secchi, and Fantuzzi (2013); and Singh, Sharma, Sutton, Hatton, and Khan (2018) has low accuracy. Therefore, current industries require a system defined for a dynamic environment that which can interact with human operations because most warehouses are still developing to automated warehouses and operations are based on a semi-automated principle (S.n. Liu, 2008) while using low-computational power for algorithm processing.

This paper aims to develop a traffic control algorithm for multiple AGV system and presents the results of this implementation for the industrial warehouse. Keeping the aim and objective of this paper, the first section presents the overview of currently proposed traffic control algorithms for multiple AGV systems for industrial warehouses in a few types of research. The second section presents the developed algorithm for multiple AGV systems and the implementation and simulation results of a developed algorithm on a real-life industrial warehouse. Finally, a discussion on the future developments and implementation of the developed traffic control algorithm in the warehouse is presented.

\section{BACKGROUND}

Supply chain management is the management of the flow of goods and services and includes all processes that transform raw materials into final products (Chopra \& Meindl, 2015; Christopher, 2016). The flow of goods in supply chain required storage and warehouse management as the most essential activities in any supply chain. Warehousing is a process of storing goods on low demand and distribute according to the demand (Myerson, 2012). To optimize the supply chain management, the efficiency of the warehousing process can be increased using warehouse management system (Christopher, 2016; Ramaa, 2012).

Over the past years, with the intensifying of the concept of Industry 4.0, the fourth industrial revolution, the world has been comprehending important progress and adoption of new advanced and disruptive technologies, such as; Robotics and Automation, 3D Printing (3DP), Artificial Intelligence (AI), Internet of Things (IoT), Drones, Business Analytics, Virtual and Augmented Reality (AR/VR) (e.g. Akbari \& Ha, 2019; Balasooriya et al., 2019; Maleesha Kulasekara, Kavalchuk, \& Smith, 2019). The vision of Industry 4.0 highlight the global machinery networks that are intellectual to exchange and share information, and monitor and control each other activities (Tjahjono et al., 2017). The fourth industrial revolution (Industry 4.0) has emerged as the promising technology to accomplish accuracy, efficiency and precision (Rajput \& Singh, 2019). The physical world and the virtual world are now bounded and connected based on the fourth industrial revolution, and the interaction between human and machines are taken places (Nascimento Daniel Luiz, 2019). This revolution facilitates the supply chain to achieve sustainability (Akbari, Clarke, Dang-Pham, \& Nkhoma, 2017) fostering the triple bottom line dimensions (i.e. Environment, Economic, and Social) (Ahi \& Searcy, 2013; Manavalan \& Jayakrishna, 2019; Schinkus, Akbari, \& Clarke, 2019).

Numerous companies are transforming or upgrading their supply chain activities towards automation, intelligence and flexibility (Akbari \& Hopkins, 2019; Mousavi, Yap, Musa, \& Dawal, 2017). Multiple AGV systems are widely used in the process of the transformation. AGVs are smart mobile vehicles controlled by computer and equipped with many guidance systems, such as optical, magnetic, laser, etc, for automation functionality (Vasava, 2014). Efficient scheduling of AGVs will result in a significant increase in productivity and reduction of operational cost (Mousavi et al., 2017; Reddy \& Rao, 2011).

Designing a Multiple AGVs system for warehouse operations should satisfy two main requirements:

- The system should manage traffic between AGVs to eliminate collisions between AGV;

- AGV system should detect changes in its environment and manage the collision between AGVs and other obstacles such as people and storage racks. 
To manage the AGV traffic in a warehouse, it needs to eliminate the collision between $\mathrm{AGVs}$ and the collision between AGV and the obstacle in the environment. There are three types of collision can be occurred in a static environment:

- Intersection collisions;

- Catching up collisions;

- Facing collisions
As seen in Figure 1, a collision occurs at an only node when two AGVs reach the same intersection at once. Additionally, catching up collision occurs when two AGV moving in the same path in the same direction but with different velocities. Facing collisions occurs due to the motion of two AGV on the same path in opposite directions.

In consideration of a dynamic environment, the obstacles of the environment keep changing with time. An AGV system should detect these environmental changes and manage collision between AGVs and the obstacles.

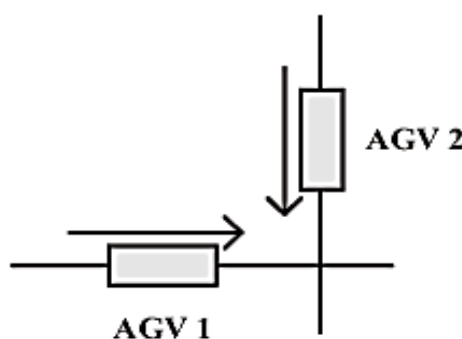

(a) Intersection Conflict

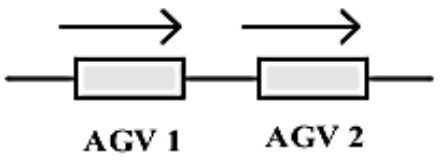

(b) Catching up with Conflict

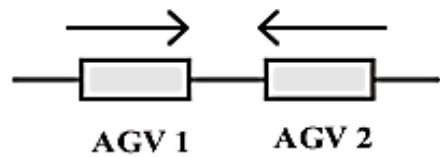

(c) Facing Conflict

Figure 1 AGV collisions types (C. Liu et al., 2017)

\section{OVERVIEW OF CURRENT ALGORITHMS}

Implementation of the traffic control algorithm is one of the fundamental design processes when initiating multiple AGVs in warehouse operations. Many pieces of research have been done to find an efficient traffic control algorithm to minimize operating costs (Yingfeng Zhang et al., 2017), moving time (Secchi et al., 2015) or Energy Efficiency (W. Zhang et al., 2018) of Multiple AGV systems.

When referring to the most proposed and implemented traffic control algorithms(Fang et al., 2018; Herrero-Perez \& Martinez-Barbera, 2010; Herrero-Perez \& Matinez-Barbera, 2008; Mazzeo \& Loiseau, 2004; Noh \& Yoon, 2016; Olmi, Secchi, \& Fantuzzi, 2011; Yi Zhang, Wang, Fu, \& Su, 2018), the traffic control process of those algorithms can be divided into 5 steps as shown below,
A. Map Modelling
B. Path planning
C. Collision identification
D. Priority planning
E. Collision avoidance

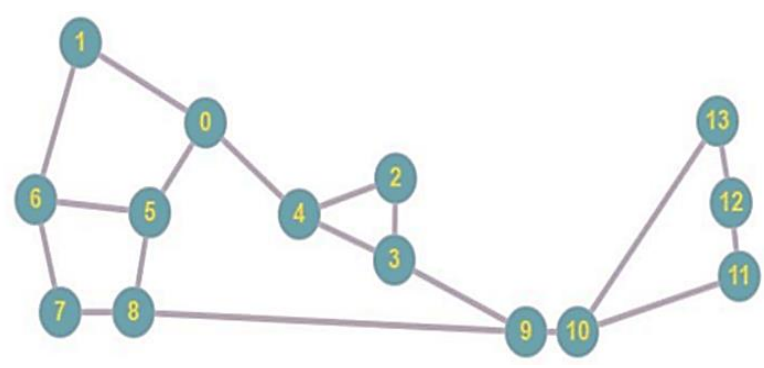

a) without weight

\subsection{Map Modelling}

When implementing the AGV system to a warehouse, it is required that the map of the warehouse is fed to the AGV system. For this, the map of the warehouse needs to be modeled according to a method that a computer system can understand mathematically. Two main methods were explained in several studies.

\subsubsection{Graph Theory (Fang et al., 2018)}

In the graph theory map is represented by a set of Nodes and Edges. More specifically, the graph of the map is presented as $\mathrm{G}=(\mathrm{N}, \mathrm{E}, \mathrm{W})$, where $\mathrm{N}$ is the set of nodes where the node is basically junction in the map and $\mathrm{E}$ is a set of edges where the edge is the path between two junctions in the map, and each edge in E connects two nodes in N. The weight of edges in $\mathrm{E}$, on the other hand, the effective distance between two nodes is presented as $\mathrm{W}_{\mathrm{ij}}$, where and $\mathrm{i}, \mathrm{j}=1,2$, ......, $\mathrm{k}$ and $\mathrm{k}$ is the number of nodes in the map. When there is no edge between given two nodes, the value of weight is set to zero. Example graph theory representation of map shown in Figure 2.

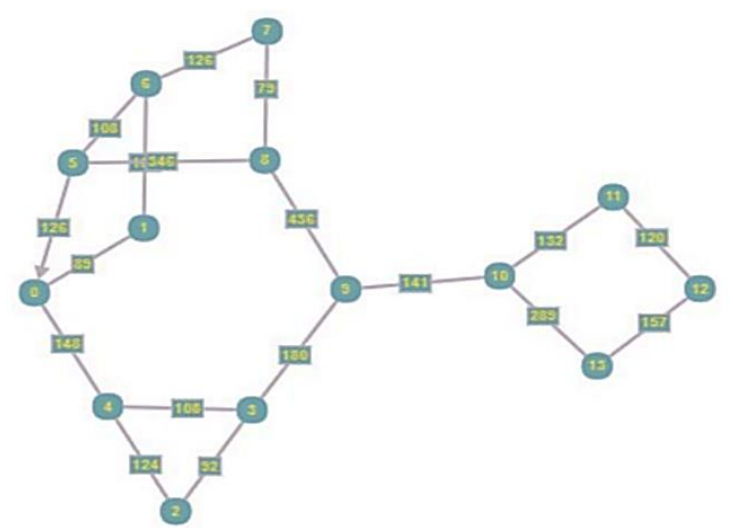

b) with weight

Figure 2 Graph theory map representation (Deepa, Kumar, Manimaran, Rajakumar, \& Krishnamoorthy, 2018) 
3.1.2 Occupancy Grid Representation (Sundram, Van Nguyen, Soh, Bouffanais, \& Wood, 2018)

In this map modeling method, a discrete grid of cells is used to represent the environment and each cell represents a certain area of the environment. This grid of cells is known as the Occupancy Grid. In this occupancy grid, all the cells are the same in size and the size of the cell is determined by the size of the autonomous vehicles which are used in the system. When the map was modeled using an occupancy grid each cell has the value or state depending on the objects in the environment. When there is a free space in the environment the state of the corresponding cell is free, otherwise, it is occupied. By this method, the states of all the cells are changed according to the environment.

In comparison, both map modelling methods are mathematically based because the implementation process in a computer system is not complex for both map modelling techniques. When comparing application scenarios of these methods occupancy grid method is mostly used to model complex environmental layouts with detailed information about the environment. However, a model of a warehouse map doesn't require to include all details about obstacles. Therefore, graph theory is sufficient to model a map for the AGV system and it only requires low computational power for processing such as path planning.

\begin{tabular}{|l|l|l|l|l|}
\hline 1 & 1 & 1 & 1 & 1 \\
\hline 1 & 0 & 1 & 0 & 1 \\
\hline 1 & 0 & 1 & 0 & 1 \\
\hline 1 & 1 & 1 & 1 & 1 \\
\hline 0 & 0 & 1 & 0 & 0 \\
\hline
\end{tabular}

\section{0 - Represent occupied cells \\ 1 - Represent free cells}

Figure 3 Occupancy grid representation (Parungao, Hein, \& Lim, 2018)

\subsection{Path Planning}

In warehouse AGV systems, paths for each AGV need to be planned according to the task demand in the warehouse. Generally, there are lots of path planning algorithms that were introduced in the robotics field such as the Dijkstra algorithm, A* algorithm, Breath first search, Depth-first search, ant colony algorithm, genetic algorithm, artificial potential field, and fuzzy path planning algorithm. However, according to several studies on the warehouse AGV systems, only the Dijkstra algorithm and $\mathrm{A}^{*}$ algorithm were used for this path planning process. Also, these algorithms were improved and discussed in these studies. A brief explanation about these algorithms and the improvements were discussed below.

\subsubsection{Dijkstra (Singh et al., 2018)}

This algorithm is based on a greedy algorithm to find the shortest path between two points. This Dijkstra algorithm can only apply on the maps which are modelled using graph theory. The pseudo-code of the pathfinding process can be shown as follows,

1. Create a cost matrix ct[V][V] from the adjacency matrix graph [V][V].

2. Array visited [] is initialized with value 0

3. If vertex 3 is source vertex then visited [3] is marked 1

4. Create a distance matrix $n$ by sorting the cost of vertices from vertex 0 to $n-1$ from source vertex 3

5. Choose a vertex $x$ such that distance $[x]$ is minimum and visited [x] is 0 mark visited [x] as 1

6. Again calculate the shortest distance of remaining vertices from the source.

\subsubsection{A* Algorithm (Lin et al., 2017)}

A* algorithm is a heuristic search algorithm based on the Dijkstra algorithm and Breath first search algorithm. This $\mathrm{A}^{*}$ algorithm can be applied on the map which is modeled using the occupancy grid method or graph theory. The process of $\mathrm{A}^{*}$ path planning algorithm can be described as follow.

The core function of the $\mathrm{A}^{*}$ algorithm is the Breath first search algorithm. A* uses a cost function to choose the node with the lowest from the nearest nodes. And cost estimation function express as follows.

$$
f(n)=g(n)+h(n)
$$

In this formula $\mathrm{f}(\mathrm{n})$ represent the total cost of the node $\mathrm{n}$ and $\mathrm{g}(\mathrm{n})$ represent the cost from starting node to current node $\mathrm{n} . \mathrm{h}(\mathrm{n})$ is a heuristic function that estimates cost from the current node $n$ to the end node. In this formula $g(n)$ calculate directly but $h(n)$ cannot be calculated in that way. For that $h(n)$ will be estimated and according to this study Manhattan distance, Chebyshev distance and Euclidean distance are the main three different methods of estimation. Since Euclidean distance is more practical it will be used in the $A^{*}$ algorithm. Euclidean distance estimation formula expressed as follows,

$$
\begin{gathered}
h(n)=D * \max (\mid n \cdot x-\text { goal. } x|+| n \cdot y-\text { goal. } y \mid) \\
D \text { represents the minimum cost between two nodes }
\end{gathered}
$$

By comparing Dijkstra and $\mathrm{A}^{*}$ algorithm, the $\mathrm{A}^{*}$ algorithm improved the shortest pathfinding algorithm based on the Dijkstra pathfinding algorithm. Therefore, the performance-wise A* algorithm has higher performance than the Dijkstra algorithm when applying on more complex map models such as occupancy grids. However, for the AGV systems, graph theory is used for map modelling and therefore, the performance of the Dijkstra algorithm is sufficient as the path planning algorithm for multiple AGV systems.

\subsection{Collision Identification}

When implementing a multiple AGV system to a warehouse, the major problem is the collisions that occur between AGVs while it's operation. These collisions need to be identified in order to avoid those. Many studies have proposed several methods of identifying collisions between 
AGVs in the simulation level before it occurs in real-time. Few of these collision identification topologies were discussed in this section.

\subsubsection{Time Window Method (Juntao, Tingting, Yuanyuan, \&} Yan, 2016)

As explained in this study, the time window method is used on the maps which modeled by the occupancy grid method. In this method, occupied time and the free time for each cell is calculated by simulating the AGV motion path.

\section{$R_{n}^{k}$ - the retention time of $n$th cell from $k$ th $A G V$ $F_{n}^{k}$ - Free time of nth cell from kth $A G V$}

From the calculation, each cell of the modeled map has some retention time from AGVs and the free times as well. If any of cell has retention time from multiple AGVs which overlaps at the same time, a conflict can be identified.

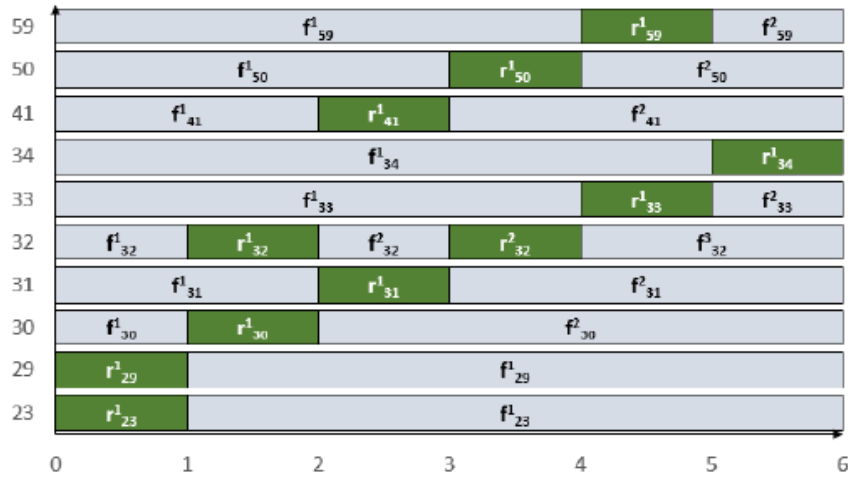

Figure 4 Time window model (Juntao et al., 2016)

\subsection{Collision Avoidance}

After collision has been identified employing a collision identification algorithm is required which several studies proposed some strategies to avoid these collisions. In most proposed multiple AGV systems, there are mainly three different collisions that can be occurred: (a) Catchup collisions, (b) Facing collisions and (c) Intersections collisions. In multiple AGV system, all AGVs has the same velocity and therefore catching up collision won't occur. The only collision which needs to be avoided is facing collision and intersection collision. And according to most studies, these two types of collisions can be avoided by the waiting strategy and re-planning the path for the AGV. These main two strategies were discussed below.

\subsubsection{Waiting Strategy (Fang et al., 2018; Lin et al., 2017)}

This collision avoiding strategy is basically, one AGV is waiting at an intersection until another AGV passes through the intersection and clear the path for the first AGV. Therefore, the waiting strategy is applied to avoid the intersection collision. But this waiting strategy cannot avoid the facing collisions.

\subsubsection{Re-planning Path (Fang et al., 2018; Lin et al., 2017)}

This AGV path Re-planning strategy is used to eliminate the facing collision between two AGVs. According to many studies facing collision is taken as an unavoidable collision type and only the solution is re-planning the path. In this technique, the alternate path will be re-planned for one AGV when it detects a collision between two AGVs. This replanning the path strategy can be applied to avoid both types of collisions but using this strategy to avoid intersection collision may reduce the efficiency of the system as the replanned path may longer than the shortest path.

\subsection{Priority Planning}

Following the collision avoidance strategies in multiple AGVs systems, each AGV should have a priority level. As an example, for the waiting strategy, one AGV should wait until another AGV is moving and the system should decide which AGV has to stop. For this system use priority level and stop the AGV with the lowest priority.

\subsubsection{Fixed Priority}

In some Multiple AGV systems, priorities for each AGV have been pre-defined when implanting the system to a warehouse.

\subsubsection{Dynamic Priority (Y. Zhang et al., 2018)}

Besides assigning fixed priorities to the AGVs some multiple AGV systems are implemented a simple algorithm to assign the priority level to each AGV depending on system state. As an example, in some AGV systems AGVs are prioritized according to the state of charge of the AGV. When a certain AGV low state of charge in its battery, the priority of this AGV will be high and it can complete its task quickly to save its energy.

By comparing these two priority assignment methods, dynamic priority assignment method can optimize the system rather than the fixed priority assignment method. According to the studies, most of the AGV systems which are already implemented are using dynamic priority assignment method and it is the most suitable priority method for Multiple AGV systems.

\section{DEVELOPED ALGORITHM}

This section presents a developed traffic control algorithm for multiple AGV systems for semi-automated warehouses. This algorithm was developed by improving existing methods discussed above.

\subsection{Map Modelling}

To manage AGVs using the automated system a model of the map is required to the system to plan the task assignment for each AGV. There are several methods of modeling maps for automated systems and in this case topological map was created using the graph theory. More specifically, the graph of the map is presented as $G=(N, E)$, where $\mathrm{N}$ is set of nodes and $\mathrm{E}$ a set of edges, and each edge in $\mathrm{E}$ connects two nodes in $\mathrm{N}$. The weight of edges in $\mathrm{E}$ are presented as $\mathrm{W}_{\mathrm{ij}}$, where $i \neq j$ and $\mathrm{i}, \mathrm{j}=1,2, \ldots ., \mathrm{k}$ and $\mathrm{k}$ is the number of nodes in the map. When there is no edge between given two nodes, the value of weight is set to zero.

\subsection{Path Planning}

Next, the path for each AGV needs to be plan depends on the task demand in the warehouse. For this path planning 
process, many algorithms can be used, such as the Dijkstra algorithm, A* algorithm, Breath first search, Depth-first search, ant colony algorithm, genetic algorithm, artificial potential field, and fuzzy path planning algorithm $(\mathrm{C}$. Liu et al., 2017). But in this proposed algorithm Dijkstra path planning algorithm was used due to its efficiency and simplicity when comparing to the alternative solutions. The planned paths can be represented as function $\mathrm{P}_{\mathrm{i}}$, where $\mathrm{i}=$ $1,2, \ldots, n$ and $n$ is the number of AGVs that operate in the warehouse. And $P_{i}$ is a set of nodes that a specific AGV needs to pass while completing its task.

\subsection{Collision Identification and Collision Avoidance}

After planning the path for each AGV according to its task collisions may occurs in a node or edge after executing the AGVs to complete its tasks. To avoid these collisions between AGVs, AGVs can drive in different velocities along its path. For this, a Velocity profile can be defined for each AGV which includes the velocities that need to be moved along each edge of its planned path. To be more specific velocity profile can present as $V_{i}$, where $i=1,2, \ldots, n$ and $n$ is the number of AGVs that operate in the warehouse. And $\mathrm{V}_{\mathrm{i}}$ is a set of velocities that a specific AGV needs travel through each edge.

The process of collision identification and velocity profile calculation procedure can be explained as follows.

- First, the maximum speed for AGVs should be defined in the system. Because of limited maximum speed, the catch-up conflict between AGVs can be avoided.

- When tasks are assigned to AGVs in the warehouse, a priority level must be assigned to each AGV due to the battery limited charge in AGVs. The priority should be based on the sate of charge of the battery in the AGVs. As an example, the AGV which has the lowest state of charge should give the heights priority and assigned the path with the lowest path length to avoid full battery discharge.

- Then a simulation must be done before executing AGVs for completing its tasks to calculate the velocity profile and to verify there no collision between AGVs when executing the task.

- In the simulation, the location of each AGV was calculated over time using a known grid and operating velocity. The pre-defined maximum velocity was used to calculate, the first iteration of each AGV's location over the time is stored. Then the calculated locations of all AGVs are used to find any conflicts between AGVs while completing their tasks. When comparing locations of two AGVs, a conflict can be found in these two AGVs that got the same location on a specific time.
At this point, the only conflict that can happen was the intersection conflict which occurred in a node. To avoid this type of collision, the velocity of $\mathrm{AGV}$ with the lowest priority (Dynamic priority planning was used) is needed to be reduced, only for the edge before the node in which collision happens. In this way, velocities need to be reduced to eliminate the collisions. After velocities were changed for the all $\mathrm{AGVs}$, the location estimation overtime for all AGVs must be recalculated and re-compared to find any conflicts. This loop must be done until there are no conflicts between each AGV in the simulation.

\subsection{AGV Collision Management on A Dynamic Environment}

Driving AGVs under different velocities on its path using a velocity profile is a strategy that only can eliminate collisions between AGVs. In the implementation process of the AGV system in a semi-automated warehouse, the AGV system should include a strategy to manage the collision between AGV and obstacles in the environment because in a semi-automated warehouse environment keeps change with time due to the human activities.

To detect the environmental changes, all the AGVs should be equipped with environmental scanning sensors such as LIDARs. When any of AGV detects an obstacle while move along its path the AGV should immediately stop to avoid collision and send data about the obstacle location to the control panel. Then the AGVs which path is blocked by obstacle should follow an alternative path which planned using a traffic control algorithm. If these AGVs don't have an alternative path to complete its task, then those AGVs should stop and notify to remove the obstacle.

\section{VALIDATION OF THE IMPLEMENTED ALGORITHM}

For an illustrative example, the proposed algorithm is simulated using MATLAB software to check its validity and performances under a real-life situation.

\subsection{Sample Problem Explanation}

For the simulation of the proposed algorithm, a real-life map of a warehouse is used.

A floorplan of a manually operated warehouse is shown in Figure 5. In this sample warehouse, a package is transferred from picking area to storage and after that, it needs to be transferred to the packing area. Due to the complexity of the tasks and variations in the goods types, multiple AGVs system is required to be implemented to significantly improve operational efficiency. 


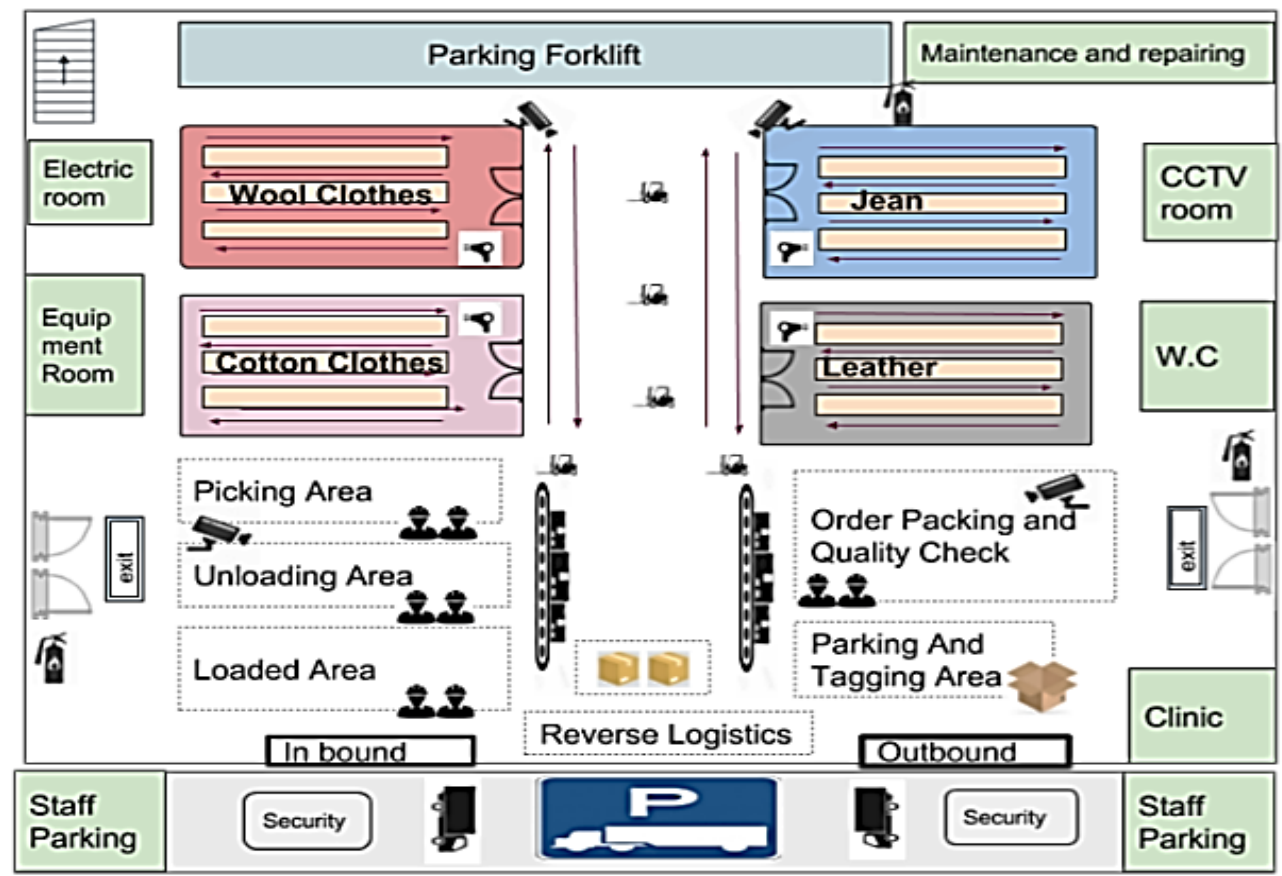

Figure 5 Plan of the sample warehouse ( $45 \mathrm{~m} \mathrm{X} \mathrm{40m)}$

\subsection{Map Modeling from Real-life Map}

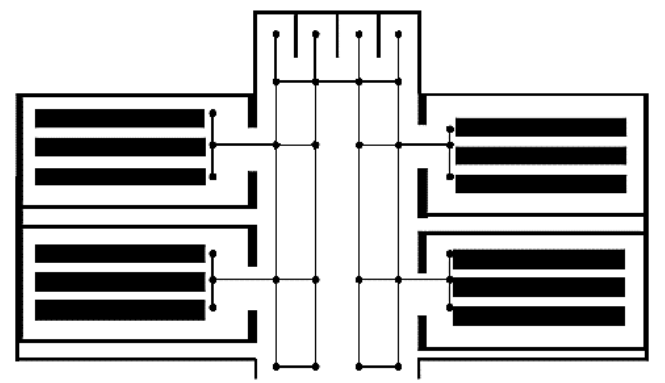

Figure 6 Node and edges representation on a map

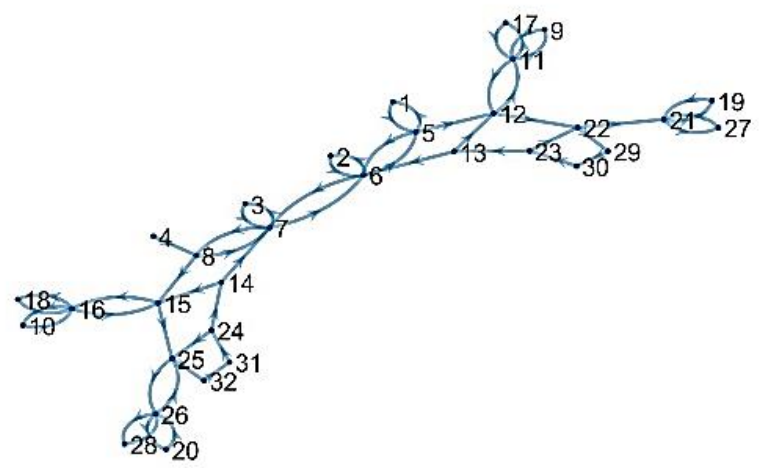

Figure 7 Graph theory representation of the map

The map of the warehouse needs to model as defined for the simulation process. This modeling process starting with recreating a binary image of the floor plan. When creating this binary image, it only included the areas that AGVs needs to move while complete its tasks. In this scenario, the AGV parking area, storage units, picking area, and Packing area were illustrated the binary image of the map.
After the illustration map needs to represented using graph theory (Error! Reference source not found.). For this, all the data about the node and edges need to define in MATLAB software. After defining data of the map, these nodes and edges can show in the illustrated map (Error! Reference source not found.).

\subsection{Path Planning}

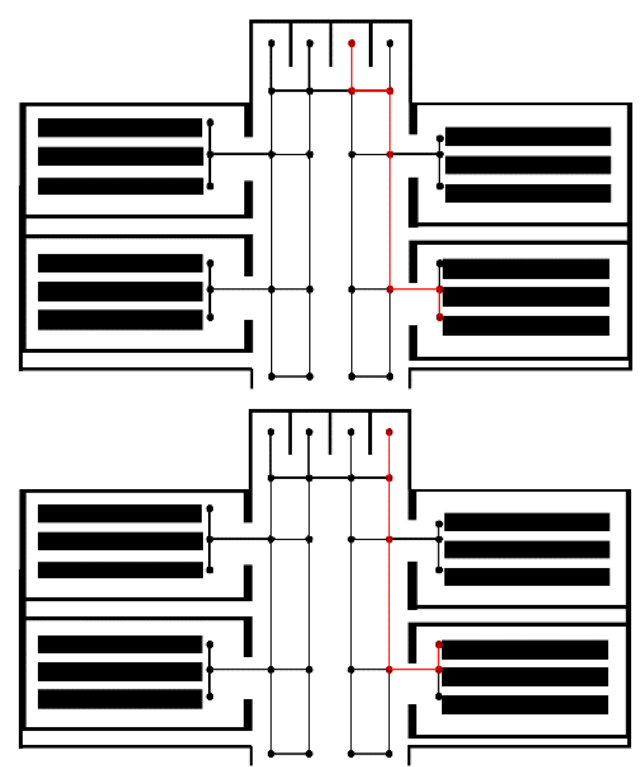

Figure 8 Planned path in simulation for two different AGVs

After representing the map using the graph theory, this model map in MATLAB can be used for the path planning process. For this path planning process, several path planning algorithms can be used such as the Dijkstra algorithm, A* algorithm, Breath first search, Depth-first search, ant colony 
algorithm, genetic algorithm, artificial potential field, and fuzzy path planning algorithm. But in this simulation Dijkstra algorithm was used due to its ease of implementation. Error! Reference source not found. shows the planned path in the MATLAB simulation from two different starting points to two different endpoints.

\subsection{Calculation and Simulation of Velocity Profile \\ After the path planning process velocity profile for each} AGV needs to be calculated according to their task assignment. The velocity profile calculation process was done according to the developed velocity profile calculation process (as described in section 4.3). The motion of AGVs with the calculated velocity profile was simulated and the location of the AGVs was plotted with respect to the time domain as seen in Figure 9. The Figure 9 warehouse floor is illustrated from the $\mathrm{X}$ and $\mathrm{Y}$ axis and the time domain is showed from the Z-axis. According to Figure 9, the plot of the motion of two AGV doesn't interest at any point until the AGVs reaches its goal location. From this plot, a conclusion can be made that developed velocity calculation algorithms calculate velocities for multiple AGV system to eliminate the collisions between AGVs.

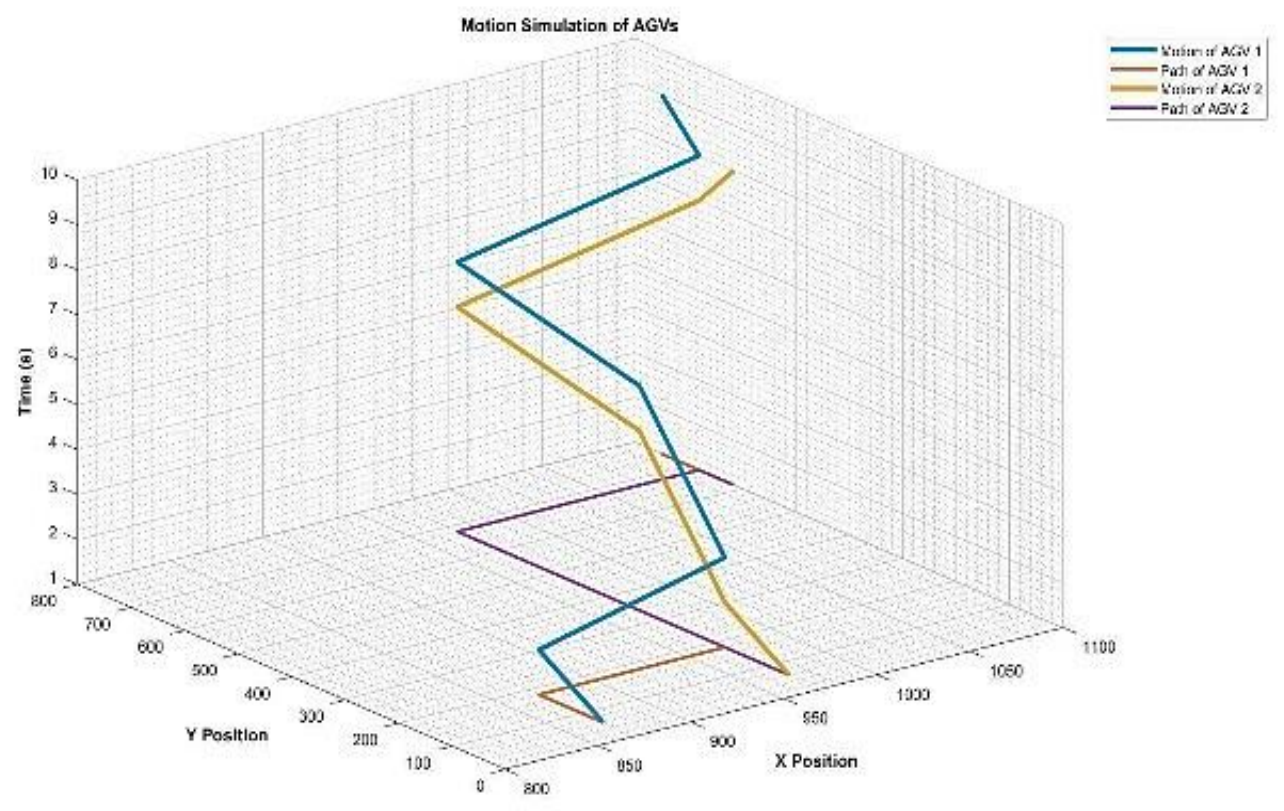

Figure 9 AGV motion simulation in the time domain

\section{CONCLUSION AND FUTUREWORKS}

In the era of technological adoption and disruption, most industrial warehouses focusing on the automation of their warehouse operations, particularly the application of multiple AGV system has the potential to transform the entire warehouse processes. Therefore, the implementation of multiple AGV system is required as a part of automation processes in warehousing. The major challenge in the implementation process of multiple $\mathrm{AGV}$ system is the managing traffic between AGVs inside the warehouse. As a solution for these, many other research proposed several traffic control algorithms and most algorithms are defined for the static environment in which human activities are not involved.

In conclusion, this paper presents a traffic control algorithm for multiple AGV system in a dynamic environment, which uses graph theory for map modeling and Dijkstra path planning algorithm for the path planning process. Then the time-based method used to simulate and calculate the optimized velocity profile for each AGV to eliminate the collision between AGVs.
This study also has some limitations which prevent the implementation of the proposed system in a practical situation. The simulations performed to validate the proposed system is carried out for a system with four AGVs and assume that all the AGVs starts its tasks at the same time. Therefore, the maximum number of AGVs that can handle by the proposed system is unknown and the performance of the system when AGV starts its task at different times also unpredictable.

As the future works of this research, the simulation of the AGV traffic control algorithm is performed for a system with a high number of AGVs and tested the performance of situations when AGV starts its task at different times. Then furthers improvements will be carried out to make the proposed system more robust. After that this multiple AGV traffic control system will implement on a live warehouse.

\section{ACKNOWLEDGMENT}

This work is successfully completed with the immense support given by the School of Science and Technology (SST) and School of Business and Management (SBM), RMIT University Vietnam, in relation to the research carried out. 


\section{REFERENCES}

Ahi, P., \& Searcy, C. (2013). A comparative literature analysis of definitions for green and sustainable supply chain management. Journal of Cleaner Production, 52, 329-341. doi:https://doi.org/10.1016/j.jclepro.2013.02.018

Akbari, M. (2013). Factors Affecting Outsourcing Decisions in Iranian Industries (Doctoral Thesis). Victoria University, Melbourne, Australia. Retrieved from http://vuir.vu.edu.au/22299/

Akbari, M., Clarke, S., Dang-Pham, D., \& Nkhoma, M. (2017). Empirical social network analysis in sustainable supply chain in Vietnam. Paper presented at the 31st Australian \& New Zealand Academy of Management (ANZAM), Melbourne, Australia.

Akbari, M., \& Ha, N. (2019). Impact of additive manufacturing on the Vietnamese transportation industry: An exploratory study. The Asian Journal of Shipping and Logistics, (In Press). doi:https://doi.org/10.1016/j.ajsl.2019.11.001

Akbari, M., \& Hopkins, J. (2016). The changing business landscape in Iran: establishing outsourcing best practices. Operations and Supply Chain Management: An International Journal, 9(3), 184-198.

Akbari, M., \& Hopkins, J. L. (2019). An investigation into anywhere working as a system for accelerating the transition of Ho Chi Minh city into a more livable city. Journal of Cleaner Production, 209, 665-679.

Balasooriya, S., Chi, T. P., \& Kavalchuk, I. (2019, 10-12 Oct. 2019). Optimized Hardware Design of Low Power and Low Cost Self Driving Multi-Purposes Robotised Platform. Paper presented at the 2019 International Symposium on Electrical and Electronics Engineering (ISEE).

Chopra, S., \& Meindl, P. (2015). Supply Chain Management: Strategy, Planning, and Operation (6th ed.). London, UL: Pearson.

Christopher, M. (2016). Logistics \& Supply Chain Management (5th ed.). UK: FT Press

Deepa, G., Kumar, P., Manimaran, A., Rajakumar, K., \& Krishnamoorthy, V. (2018). Dijkstra Algorithm Application: Shortest Distance between Buildings. International Journal of Engineering \& Technology, 7(4.10), 974-976.

Digani, V., Sabattini, L., Secchi, C., \& Fantuzzi, C. (2013). Towards decentralized coordination of multi robot systems in industrial environments: A hierarchical traffic control strategy. Paper presented at the 2013 IEEE 9th International Conference on Intelligent Computer Communication and Processing (ICCP).

Fang, J., Xiaolong, L., Jichao, W., Yunde, S., Fengyu, X., \& Zhang, Z. (2018). Research on Multi-AGV Autonomous Obstacle Avoidance Strategy Based on Improved A* Algorithm. Paper presented at the 2018 25th International Conference on Mechatronics and Machine Vision in Practice (M2VIP).

Herrero-Perez, D., \& Martinez-Barbera, H. (2010). Modeling distributed transportation systems composed of flexible automated guided vehicles in flexible manufacturing systems. IEEE Transactions on Industrial Informatics, 6(2), 166-180.

Herrero-Perez, D., \& Matinez-Barbera, H. (2008). Decentralized coordination of autonomous agvs in flexible manufacturing systems. Paper presented at the 2008 IEEE/RSJ International Conference on Intelligent Robots and Systems.

Jazdi, N. (2016, 19-21 May 2016). Dynamic calculation of the reliability of factory automation applications: Industry 4.0 applications. Paper presented at the 2016 IEEE International Conference on Automation, Quality and Testing, Robotics (AQTR).

Juntao, L., Tingting, D., Yuanyuan, L., \& Yan, H. (2016). Study on robot path collision avoidance planning based on the improved ant colony algorithm. Paper presented at the 2016 8th International Conference on Intelligent Human-Machine Systems and Cybernetics (IHMSC).

Kasemset, C., \& Sudphan, J. (2014, 9-12 Dec. 2014). Warehouse storage assignment: The case study of a plastic bag manufacturer. Paper presented at the 2014 IEEE International Conference on Industrial Engineering and Engineering Management.

König, A., \& Spinler, S. (2016). The effect of logistics outsourcing on the supply chain vulnerability of shippers: Development of a conceptual risk management framework. The International Journal of Logistics Management, 27(1), 122-141. doi:doi:10.1108/IJLM-03-2014-0043

Lin, M., Yuan, K., Shi, C., \& Wang, Y. (2017). Path planning of mobile robot based on improved $A *$ algorithm. Paper presented at the 2017 29th Chinese Control And Decision Conference (CCDC).

Liu, C., Tan, J., Zhao, H., Li, Y., \& Bai, X. (2017). Path planning and intelligent scheduling of multi-AGV systems in workshop. Paper presented at the 2017 36th Chinese Control Conference (CCC).

Liu, S.-n. (2008, 12-15 Oct. 2008). Optimization problem for AGV in automated warehouse system. Paper presented at the 2008 IEEE International Conference on Service Operations and Logistics, and Informatics.

Maleesha Kulasekara, V., Kavalchuk, I., \& Smith, A. (2019, 20-21 July 2019). Smart Key System Design for Electric Bike for Vietnam Environment. Paper presented at the 2019 International Conference on System Science and Engineering (ICSSE).

Manavalan, E., \& Jayakrishna, K. (2019). An Analysis on Sustainable Supply Chain for Circular Economy. Procedia Manufacturing, 33, 477-484. doi:https://doi.org/10.1016/j.promfg.2019.04.059

Mazzeo, S., \& Loiseau, I. (2004). An ant colony algorithm for the capacitated vehicle routing. Electronic Notes in Discrete Mathematics, 18, 181-186.

Mousavi, M., Yap, H. J., Musa, S. N., \& Dawal, S. Z. M. (2017). A Fuzzy Hybrid GA-PSO Algorithm for Multi-Objective AGV Scheduling in FMS. International Journal of Simulation Modelling, 16(1), 58-71.

Mujkić, Z., Qorri, A., \& Kraslawksi, A. (2018). Sustainability and Optimization of Supply Chains: a Literature Review. Operations and Supply Chain Management: An International Journal, 11(4), 186-199.

Myerson, P. (2012). Lean supply chain and logistics management. New York, USA: Mc Graw Hill Education.

Nascimento Daniel Luiz, M. (2019). Exploring Industry 4.0 technologies to enable circular economy practices in a manufacturing context: A business model proposal. Journal of Manufacturing Technology Management, 30(3), 607-627. doi:10.1108/JMTM-03-2018-0071

Noh, Y., \& Yoon, Y. (2016). Tactical traffic control for multiple $A G V$ systems based on three dimensional space. Paper presented at the AIP Conference Proceedings.

Oleari, F., Magnani, M., Ronzoni, D., \& Sabattini, L. (2014, 4-6 Sept. 2014). Industrial AGVs: Toward a pervasive diffusion in modern factory warehouses. Paper presented at the 2014 IEEE 10th International Conference on Intelligent Computer Communication and Processing (ICCP).

Olmi, R., Secchi, C., \& Fantuzzi, C. (2011). An efficient control strategy for the traffic coordination of AGVs. Paper presented at the 2011 IEEE/RSJ International Conference on Intelligent Robots and Systems.

Park, S., Crespi, N., Park, H., \& Kim, S. (2014, 6-8 March 2014). IoT routing architecture with autonomous systems of things. Paper presented at the 2014 IEEE World Forum on Internet of Things (WF-IoT) 
Parungao, L., Hein, F., \& Lim, W. (2018). Dijkstra algorithm based intelligent path planning with topological map and wireless communication. ARPN Journal of Engineering and Applied Sciences, 13(8), 2753-2763.

Petrinec, K., Kovacic, Z., \& Marozin, A. (2003, 10-12 Dec. 2003). Simulator of multi-AGV robotic industrial environments. Paper presented at the IEEE International Conference on Industrial Technology, 2003.

Rajput, S., \& Singh, S. (2019). Industry 4.0 - challenges to implement circular economy. Benchmarking: $A n$ International Journal, (In Press).

Ramaa, A. S., K.N.; Rangaswamy, T.M. (2012). Impact of Warehouse Management System in a Supply Chain. International Journal of Computer Applications, 54(1), 1420.

Reddy, B. S., \& Rao, C. S. P. (2011). Flexible manufacturing systems modelling and performance evaluation using Automod. International Journal of Simulation Modelling, 10(2), 78-90.

Robotics Business Review. (2019). LogisticsIQ: Warehouse Automation Market to Hit $\$ 27 \mathrm{~B}$ by 2025 . Retrieved from https://www.roboticsbusinessreview.com/news/reportwarehouse-automation-logisticsiq/

Schinkus, C., Akbari, M., \& Clarke, S. (2019). Corporate Social Responsibility in Sustainable Supply Chain Management: An Econo-Bibliometric Perspective. Theoretical Economics Letters, 9, 247-270. doi:10.4236/tel.2019.91020

Secchi, C., Olmi, R., Rocchi, F., \& Fantuzzi, C. (2015, 26-30 May 2015). A dynamic routing strategy for the traffic control of AGVs in automatic warehouses. Paper presented at the 2015 IEEE International Conference on Robotics and Automation (ICRA).

Singh, Y., Sharma, S., Sutton, R., Hatton, D., \& Khan, A. (2018, 25-27 April 2018). Feasibility study of a constrained Dijkstra approach for optimal path planning of an unmanned surface vehicle in a dynamic maritime environment. Paper presented at the 2018 IEEE International Conference on Autonomous Robot Systems and Competitions (ICARSC).

Smolic-Rocak, N., Bogdan, S., Kovacic, Z., \& Petrovic, T. (2010). Time Windows Based Dynamic Routing in Multi-AGV Systems. IEEE Transactions on Automation Science and Engineering, 7(1), 151-155. doi:10.1109/TASE.2009.2016350

Sundram, J., Van Nguyen, D., Soh, G. S., Bouffanais, R., \& Wood, K. (2018). Development of a Miniature Robot for Multi-robot Occupancy Grid Mapping. Paper presented at the 2018 3rd International Conference on Advanced Robotics and Mechatronics (ICARM).

Tjahjono, B., Esplugues, C., Ares, E., \& Pelaez, G. (2017). What does Industry 4.0 mean to Supply Chain? Procedia Manufacturing, $\quad 13, \quad 1175-1182$. doi:10.1016/j.promfg.2017.09.191

Vasava, A. S. (2014). Scheduling of automated guided vehicle in different flexible manufacturing system environment. International Journal of Innovative Research in Advances Engineering, 1(8), 262-267.

Zhang, W., Peng, Y., Wei, W., \& Kou, L. (2018). Real-Time Conflict-Free Task Assignment and Path Planning of MultiAGV System in Intelligent Warehousing. Paper presented at the 2018 37th Chinese Control Conference (CCC).

Zhang, Y., Wang, F., Fu, F., \& Su, Z. (2018). Multi-AGV Path Planning for Indoor Factory by Using Prioritized Planning and Improved Ant Algorithm. Journal of Engineering and Technological Sciences, 50(4), 534-547.

Zhang, Y., Zhu, Z., \& Lv, J. (2017). CPS-based smart control model for shopfloor material handling. IEEE Transactions on Industrial Informatics, 14(4), 1764-1775.

Zhang, Z., Guo, Q., Chen, J., \& Yuan, P. (2018). Collision-Free Route Planning for Multiple AGVs in an Automated Warehouse Based on Collision Classification. IEEE Access, 6, 26022-26035. doi:10.1109/ACCESS.2018.2819199

Mr. Pasan Luchmantha Dharmasiri is an undergraduate student in Robotics and Mechatronics Engineering at School of Science and Technology (SST), RMIT University, Vietnam. He has worked at two industrial automation companies and involved with several industrial automation projects. And also, he published few conference papers on the implantation process of multiple AGV systems in the industrial warehouses within the time periods of 2019 to 2020 . His research interests include robotics in industrial automation and wireless communication.

Dr. Ilya Kavalchuk is head of Hyperspeed Transportation Complex department at Unitsky Sting Technologies Co. He got his $\mathrm{PhD}$ from RMIT University, Australia. His research interest includes Sustainabvle Transportation systems, advanced control systems, communication technologies development and engineering education.

Dr. Mohammadreza Akbari is a Senior Lecturer and Program Manager (Logistics \& Supply Chain Management) in the School of Business and Management (SCM), RMIT University, Vietnam. He graduated from Victoria University in Australia with a Doctor of Business Administration (DBA), in Logistics and Supply Chain Management. His research interest includes a broad range of disciplines including; logistics and supply chain management, integrated, reverse and green logistics, operations management, strategic outsourcing, emerging technologies, sustainable development, corporate social responsibility (CSR), pollution reduction, anywhere working, and smart city. 